\title{
Prognostic impact of Additional Chromosomal Abnormalities in Egyptian Chronic Myeloid Leukemia Patients
}

\author{
Yasser H. EINahass ${ }^{1}$, Magda M. Assem ${ }^{1}$, Magdy M. Saber ${ }^{2}$, Sarah K. Abdalla, ${ }^{1, a \star}$, Hossam K. Mahmoud ${ }^{2}$, Fatma A. EIRefaey ${ }^{1}$ \\ ${ }^{1}$ Department of Clinical Pathology, National Cancer Institute, Cairo University, Fom El Khalig square - El Manial, Cairo, Egypt; \\ ${ }^{2}$ Department of Medical Oncology, National Cancer Institute, Cairo University, Fom El Khalig square - El Manial, Cairo, Egypt \\ ${ }^{a}$ Present address: Moffitt Cancer Center, 12902 USF Magnolia Drive, Tampa, FL 33612 - USA.
}

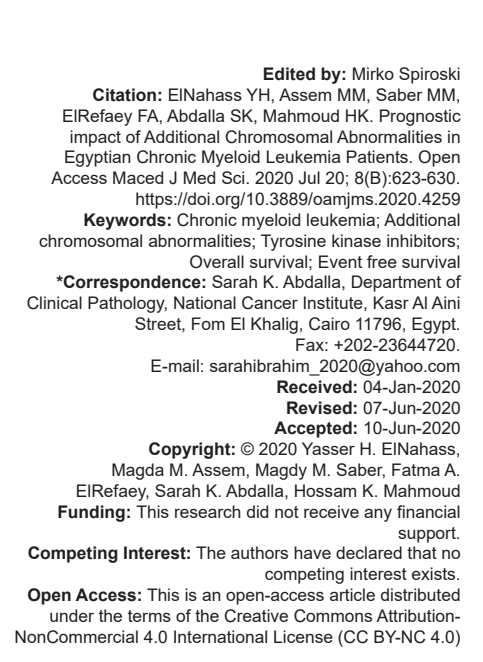

Abstract

BACKGROUND: Emergence of additional chromosomal abnormalities (ACAs) in chronic myeloid leukemia (CML) is associated with disease progression to advanced phases and reflects the genetic instability of CML.

AIM: Is to evaluate the frequency of ACAs in chronic phase (CP) and advanced disease (AP) CML patients and study their impact on patient's outcome, overall survival (OS) and event-free survival (EFS).

RESULTS: The studied group $(n=73)$ included 31 males $(43 \%)$ and 42 females $(57 \%)$. Median age of patients at diagnosis was 37 years (17-76). Median TLC was $208 \times 109 / \mathrm{L}(2.1-784.2)$, median Hb was $9.4 \mathrm{~g} / \mathrm{dL}(5.7-13)$, and median platelets count was $290.5 \times 109 / \mathrm{L}$ (13-1271). We identified 32 patients (44\%) with ACAs. ACAs emergence was significantly associated with advanced phases of CML $(13 / 21,62 \%)$ compared to CP $(19 / 52,36 \%)(p=0.048)$. ACAs were associated with lower median OS and EFS in CP compared to AP (38 vs. $120 \mathrm{~ms}$ ) and (58.3 vs. $77 \mathrm{~ms}$ ) ( $p=0.026$ and $p=0.065$, respectively). Early molecular responders $(6 / 17,35 \%)$ at 3 months, and 6 months $(10 / 26,38 \%)$ developed ACAs less than nonoptimal responders. Disease phase, hepatomegaly and bone marrow eosinophilia were significant predictors of OS ( $p<0.001, p=0.02, p=0.04$, respectively).

CONCLUSION: Early identification of ACAs in $\mathrm{Ph}+$ metaphases at diagnosis and during therapy predicts $\mathrm{CML}$ outcome. ACAs emergence occurred at a higher frequency and at a younger age in our CML patients and are related to inferior EFS and OS.

\section{Introduction}

Chronic myeloid leukemia $(\mathrm{CML})$ is a clonal myeloproliferative neoplasm characterized by the presence of the Philadelphia (Ph) chromosome, produced by the reciprocal translocation $t(9 ; 22)$ (q34;q11) [1]. This translocation leads to the generation of the chimeric fusion gene $B C R-A B L 1$ [2]. At diagnosis, additional chromosomal abnormalities (ACAs) in $\mathrm{Ph}+$ cells may appear in $\sim 5 \%$ of patients. The proportion of patients with ACAs rises during the course of the disease to $\sim 80 \%$ in the blast phase (BP), according to several series [3], [4]. Despite the efficacy of imatinib mesylate (IM) in CML - chronic phase (CP), treatment failure, or suboptimal response have been reported [5], [6]. The appearance of ACAs during tyrosine kinase inhibitor (TKI) treatment is commonly known as clonal evolution (CE) and seems to play an important role in IM resistance and is considered to be a poor prognostic feature [7]. The World Health Organization (WHO) suggests that $C M L$ patients showing ACAs emerging during treatment should be considered in accelerated phase (AP) [8] and European Leukemia Network (ELN) recommendations suggest that the presence of ACAs at diagnosis may represent a "warning" feature, requiring careful monitoring of the patient. Emerging ACAs during the course of treatment represent the failure of treatment [9], [10]. The aim of the current study is to investigate the frequency and prognostic impact of ACAs in a cohort of Egyptian CML patients, either at diagnosis or during the disease course, and to study their impact on response to TKI therapy and CML patient's survival.

\section{Patients and Methods}

\section{Patients}

This study was conducted on $73 \mathrm{CML}$ Egyptian patients either newly diagnosed or under TKI treatment who presented to the outpatient clinic of Medical Oncology Department of National Cancer Institute from 
January 2015 to January 2017. Median follow-up time was $24 \mathrm{~ms}$ (1-121 ms). Informed consent was provided by all included subjects and the study was approved by the Institutional Review Board. Eligible patients were $>18$ years fulfilling morphologic and cytogenetic criteria of $\mathrm{Ph}+\mathrm{CML}$ in $(\mathrm{CP}),(\mathrm{AP})$, or (BP) according to definitions in the revised $4^{\text {th }}$ edition of the $\mathrm{WHO}$ classification [8].

\section{Treatment}

The included CML patients who were previously treated with a TKI received one or more of either a first-generation TKI (IM, IM/ GLIVEC ${ }^{\circledR}, 400$ mg orally once per day or generic imatinib) or a secondgeneration TKI (Nilotinib/TASIGNA ${ }^{\circledR}$ or Dasatinib/ SPRYCYL ${ }^{\circledR}$ ). Hydroxycarbamide (1-6 g/day orally) was given to $C M L-C P$ patients as initial cytoreductive therapy until $B C R-A B L 1$ results by fluorescence in situ hybridization (FISH) were available.

\section{Definition of responses to TKI treatment}

Hematologic, molecular, and cytogenetic remissions were defined according to the National Comprehensive Cancer Network guidelines [11]. Definitions of molecular response were defined according to ELN recommendations [12].

\section{Measurements of survival}

Event free survival (EFS) was defined as loss of hematologic or cytogenetic response or development of advanced phases of CML (AP or BP) and overall survival (OS) by absence of death from any reason [13].

\section{Methodology}

Cultivated bone marrow (BM) samples of all included patients were subjected to chromosomal banding analysis to detect the presence of ACAs in $\mathrm{Ph}+$ cells. ACAs were classified as major route abnormalities that included: +8 , a second Philadelphia chromosome (der 22), +19, i(17q10) [4], and minor route abnormalities that included: $+21,-7,-17$, del $(Y)$ and $3 q 26.2$, and other abnormalities [9]. The detected chromosomal abnormalities were confirmed using FISH molecular probes provided by Cytocell (UK) and MetaSystems (Germany). Diagnostic and serial measurements of $B C R-A B L 1$ fusion gene mRNA transcript level were done by real-time quantitative polymerase chain reaction according to IS on GeneXpert, Cepheid.

\section{Conventional cytogenetics study}

At least 20 metaphases were analyzed for each sample after trypsinization and staining slides with Giemsa. Metaphase capture and image analysis were performed using Metafer 4/MetaClient software analysis program (MetaSystems) and CytoVision (Applied Imaging). Chromosomal abnormalities were described according to the International System for Human Cytogenetics Nomenclature (ISCN, 2016) [14].

\section{$\mathrm{FISH}$}

The following FISH probes were used: BCR/ABL translocation (Dual Fusion Probe), 17p subtelomere specific probe, $17 q$ subtelomere specific probe, Del (20q) deletion probe and EVI1 (MECOM) break apart probe (Provided by Cytocell Aquarius) and XCP (XCyting Chromosome Paints) for chromosome 8, chromosome 19, chromosome 7 , chromosome 21, and Del (7q) (q22q31) (MetaSystems Probes $\mathrm{GmbH}$ 1.Industriestr.7).

\section{Statistical methodology}

Statistical analysis was done using IBM SPSS $^{\circledR}$ Statistics version 22 (IBM ${ }^{\circledR}$ Corp., Armonk, NY, USA). Numerical data were expressed as mean and standard deviation or median and range as appropriate. Qualitative data were expressed as frequency and percentage. Pearson's Chi-square or Fisher's exact tests were used to examine the relationship between qualitative variables. For not normally distributed quantitative data, a comparison between the two groups was done using the Mann-Whitney test (nonparametric t-test). Survival analysis was done using the Kaplan-Meier method and a comparison between two survival curves was done using the log-rank test. Multivariate analysis was done using the Coxregression method for the factors affecting survival on univariate analysis. Hazard ratio (HR) with its $95 \%$ confidence interval $(\mathrm{Cl})$ was used for risk estimation. All tests were two-tailed. A $p<0.05$ was considered statistically significant.

\section{Results}

Seventy-three CML patients were included, $31(43 \%)$ males and $42(57 \%)$ females. Fifty-two/73 (71\%) patients were in CP, 6(8\%) patients were in AP, and $15(20 \%)$ patients were in BP. Patients with AP and BP were grouped together as advanced phase disease $(21,29 \%)$. The median age of CP patients at diagnosis was 39 years (17-76) and 35 years (22-54) for AP. Median TLC was 225×109/L (2.1-784.2) for CP patients and $127 \times 109 / \mathrm{L}(13.5-353)$ for $\mathrm{AP}$, median $\mathrm{Hb}$ was $10 \mathrm{~g} / \mathrm{dL}(5.7-13)$ for $\mathrm{CP}$ and $8.8 \mathrm{~g} / \mathrm{dL}(7.1-11.5)$ for AP, and median platelets count was $319 \times 109 / \mathrm{L}$ (123-1173) for CP and 237.5×109/L (13-1271) for 
AP. Median PB blast $\%$ was $2 \%(0-9)$ for $\mathrm{CP}$ and $6 \%(0-92)$ for AP, median PB eosinophils was $3 \%$ $(0-8)$ for CP and $2 \%(0-13)$ for AP, and median PB basophils was $3 \%(0-12)$ for $\mathrm{CP}$ and $3 \%(0-23)$ for AP. Median BM blast $\%$ was $2 \%(0-8)$ for $\mathrm{CP}$ and $25 \%$ (10-91) for AP, median BM basophils was $4 \%$ $(0-11)$ for $\mathrm{CP}$ and $5 \%(0-17)$ for AP, and median BM eosinophils was $5 \%(0-14)$ for CP and $4 \%(0-16)$ for AP. Time to TKI treatment ranged from 6 to 150 days, with a median of 33 days. Sixty-eight patients $(93 \%)$ were treated with $1^{\text {st }}$ line TKI upfront $\left(\right.$ Gleevec ${ }^{\circledR}$ or generic IM) and 5 patients $(7 \%)$ received a $2^{\text {nd }}$ line TKI as upfront therapy (Nilotinib/Tasigna ${ }^{\circledR}$ or Dasatinib/ Sprycel $\left.^{\circledR}\right)$. Forty three/52 $(83 \%)$ were tolerant to TKI therapy, while 9 patients $(17 \%)$ were intolerant. Ten/52 (19\%) CP and $17 / 21$ (81\%) AP patients died by the end of study duration. Cumulative OS of CP patients at 12 months was $90 \%$ and at 24 months was $84 \%$ and was $71 \%$ and $42 \%$ for AP patients at 12 months and 24 months, respectively $(p<0.001)$. EFS of CP patients $(38 / 52)$ was $73 \%$ by the end of 24 months follow-up duration.

\section{Evaluation for emergence of ACAs}

Thirty two/73 (44\%) patients developed ACAs; 22 at diagnosis ( $16 \mathrm{CP}$ and $6 \mathrm{AP}$ patients) and 10 patients later during the course of the disease $(3 \mathrm{CP}$ and $7 \mathrm{AP}$ patients). The reported 32 ACAs were 18 (56\%) major route abnormalities, $4(12 \%)$ minor route abnormalities, and $5(16 \%)$ patients with more than one ACAs (major and minor) and 5 patients (16\%) were found to express other (non-major/non-minor) secondary chromosomal changes. Ten/18 (56\%) patients developed major route abnormalities at diagnosis and $8 / 18(44 \%)$ patients during the course of disease, while $2 / 5(40 \%)$ patients developed both major and minor route abnormalities during the course of disease and $3 / 5(60 \%)$ at diagnosis. Minor route abnormalities were detected in 4 patients at diagnosis, while other chromosomal abnormalities were detected in 5 patients at diagnosis. Four/5 AP patients were identified with hyperdiploid karyotype and developed acute lymphoblastic leukemia BP and one patient developed mixed phenotype acute leukemia during disease course Table 1 describes ACAs in 32 CML patients.

Table 1: ACAs emergence in 32/73 CML patients

\begin{tabular}{|c|c|c|c|c|c|c|c|}
\hline UPN & Age & Gender & Phase & Time of emergence & Karyotype & Major/Minor/others & TKI upfront \\
\hline 3 & 22 & $\mathrm{~F}$ & $\mathrm{ABC}$ & During course & $\begin{array}{l}51, \mathrm{XX},+8,+9,+10,+19,+22, \operatorname{der}(22), \mathrm{t}(9 ; 22)(\mathrm{q} 34 ; \mathrm{q} 11) \\
{[16] / 47,2 \mathrm{q}+\mathrm{t}(9 ; 22)(\mathrm{q} 34 ; \mathrm{q} 11)[4]}\end{array}$ & Major & $1^{\text {st }}$ line \\
\hline 6 & 34 & M & $A B C$ & During course & $\begin{array}{l}52, \mathrm{XY},+8,+10,+11,11 \mathrm{q}-,+14,+21,+22, \operatorname{der}(22), \mathrm{t}(9 ; 22)(\mathrm{q} 34 ; \mathrm{q} 11) \\
{[10] / 46, \mathrm{XY}, \mathrm{t}(9 ; 22)(\mathrm{q} 34 ; \mathrm{q} 11)[10]}\end{array}$ & Major & $1^{\text {st }}$ line \\
\hline 8 & 54 & $\mathrm{~F}$ & $A B C$ & At diagnosis & $45, X X,-7, t(9 ; 22)(q 34 ; q 11)[4] / 46, X X, t(9 ; 22)(q 34 ; q 11)[16]$ & Minor & $1^{\text {st }}$ line \\
\hline 9 & 26 & $\mathrm{~F}$ & $\mathrm{CP}$ & At diagnosis & $\begin{array}{l}46, X X, t(9 ; 22)(q 34 ; q 11)[10] / 44, X X,-10,-15,-20,+22, t(9 ; 22) \\
(q 34 ; q 11)[10]\end{array}$ & Minor & $1^{\text {st }}$ line \\
\hline 10 & 58 & $\mathrm{~F}$ & $\mathrm{CP}$ & During course & $\begin{array}{l}48, X X,+19,+22, \operatorname{der}(22), t(9 ; 22)(q 34 ; q 11)[4] / 47, X X, \operatorname{der}(22), \\
t(9 ; 22)(q 34 ; q 11)[6] / 46, X X t(9 ; 22)(q 34 ; q 11)[10]\end{array}$ & Major & $1^{\text {st }}$ line \\
\hline 11 & 38 & $\mathrm{~F}$ & $\mathrm{CP}$ & At diagnosis & $\begin{array}{l}48, X X,+8,+22, \operatorname{der}(22), t(9 ; 22)(q 34 ; q 11)[8] / 47, X X,+19, t(9 ; 22) \\
(q 34 ; q 11)[6] / 46, X X, t(9 ; 22)(q 34 ; q 11)[6]\end{array}$ & Major & $1^{\text {st }}$ line \\
\hline 13 & 33 & $\mathrm{~F}$ & $\mathrm{CP}$ & At diagnosis & $46, \mathrm{XX}, \mathrm{t}(9 ; 22)(\mathrm{q} 34 ; \mathrm{q} 11)[3] / 46, \mathrm{XX},-7,+17[7] / 45, \mathrm{XX},-7[10]$ & Minor & $1^{\text {st }}$ line \\
\hline 16 & 27 & $\mathrm{~F}$ & $\mathrm{CP}$ & At diagnosis & $\begin{array}{l}47, X X,-2,+8,+19, t(9 ; 22)(q 34 ; q 22)[4] / 46, X X \\
t(9 ; 22)(q 34 ; q 22)[16]\end{array}$ & Major & $1^{\text {st }}$ line \\
\hline 18 & 26 & M & $\mathrm{CP}$ & At diagnosis & $\begin{array}{l}\text { 44, X, del }(y),-17, \mathrm{t}(9 ; 22)(\mathrm{q} 34 ; \mathrm{q} 11)[8] / 46, \mathrm{XY} \\
\mathrm{t}(9 ; 22)(\mathrm{q} 34 ; \mathrm{q} 11)[12]\end{array}$ & Minor & $1^{\text {st }}$ line \\
\hline 19 & 32 & $\mathrm{~F}$ & $A B C$ & At diagnosis & $47, \mathrm{XX},+22, \operatorname{der}(22), \mathrm{t}(9 ; 22)(\mathrm{q} 34 ; \mathrm{q} 11)[18] / 46, \mathrm{XX}[2]$ & Major & $1^{\text {st }}$ line \\
\hline 20 & 23 & $\mathrm{~F}$ & $\mathrm{CP}$ & At diagnosis & $\begin{array}{l}47, \mathrm{XX},+22, \operatorname{der}(22), \mathrm{t}(9 ; 22)(\mathrm{q} 34 ; \mathrm{q} 11)[3] / 46, \mathrm{XX}, \mathrm{t}(9 ; 22) \\
(\mathrm{q} 34 ; \mathrm{q} 11)[17]\end{array}$ & Major & $1^{\text {st }}$ line \\
\hline 27 & 40 & M & AP & During course & $47, X Y,+8,11 q-, t(9 ; 22)(q 34 ; q 11)[15] / 46, X Y, t(9 ; 22)(q 34 ; q 11)[5]$ & Major & $1^{\text {st }}$ line \\
\hline 39 & 26 & M & $A B C$ & During course & $\begin{array}{l}\text { 47, XY, +8, del }(6 q), t(9 ; 22)(q 34 ; q 11)[15] / 46, X Y, t(9 ; 22) \\
(q 34 ; q 11)[5]\end{array}$ & Major & $1^{\text {st }}$ line \\
\hline 40 & 31 & M & $\mathrm{CP}$ & At diagnosis & $45, \mathrm{XY},-9, \mathrm{t}(9 ; 22)(\mathrm{q} 34 ; \mathrm{q} 11)[14] / 46, \mathrm{XY}, \mathrm{t}(9 ; 22)(\mathrm{q} 34 ; \mathrm{q} 11)[6]$ & Other & $1^{\text {st }}$ line \\
\hline 41 & 35 & $\mathrm{~F}$ & $A B C$ & At diagnosis & $\begin{array}{l}47, X X,+21, t(8 ; 21)(q 22 ; q 22), t(9 ; 22)(q 34 ; q 11)[15] / 46, X X \\
t(9 ; 22)(q 34 ; q 11)[5]\end{array}$ & Other & $1^{\text {st }}$ line \\
\hline 42 & 18 & M & $\mathrm{CP}$ & At diagnosis & $47, \mathrm{XY},+8, \mathrm{t}(9 ; 22)(\mathrm{q} 34 ; \mathrm{q} 11)[16] / 46, \mathrm{XY}, \mathrm{t}(9 ; 22)(\mathrm{q} 34 ; \mathrm{q} 11)[4]$ & Major & $1^{\text {st }}$ line \\
\hline 43 & 26 & $\mathrm{~F}$ & $\mathrm{CP}$ & At diagnosis & $46, X X, \operatorname{der}(1 q), t(9 ; 22)(q 34 ; q 11)[20]$ & Other & $1^{\text {st }}$ line \\
\hline 45 & 23 & M & $\mathrm{CP}$ & At diagnosis & $\begin{array}{l}\text { 48, XY,+8, der (22), t(9;22)(q34;q11)[18]/46, } \\
X Y, t(9 ; 22)(q 34 ; q 11)[2]\end{array}$ & Major & $1^{\text {st }}$ line \\
\hline 46 & 48 & M & $\mathrm{CP}$ & During course & $\begin{array}{l}47, \mathrm{XY},+4, \mathrm{t}(9 ; 22)(\mathrm{q} 34 ; \mathrm{q} 11)[6] / 47, \mathrm{XY},+8, \mathrm{t}(9 ; 22)(\mathrm{q} 34 ; \mathrm{q} 11)[8] / 47 \\
\mathrm{XY}, \operatorname{der}(22), \mathrm{t}(9 ; 22)(\mathrm{q} 34 ; \mathrm{q} 11)[18]\end{array}$ & Major & $1^{\text {st }}$ line \\
\hline 50 & 56 & $\mathrm{~F}$ & $\mathrm{CP}$ & At diagnosis & $\begin{array}{l}47, X X,+22, \operatorname{der}(22), t(9 ; 22)(q 34 ; q 11)[6] / 47, X X,+19, t(9 ; 22) \\
(q 34 ; q 11)[4] / 46, X X, t(9 ; 22)(q 34 ; q 11)[10]\end{array}$ & Major & $1^{\text {st }}$ line \\
\hline 51 & 29 & M & $A B C$ & During course & $\begin{array}{l}48, X Y,+8,+22, \operatorname{der}(22), t(9 ; 22)(q 34 ; q 11)[16] / 46, X Y, t(9 ; 22) \\
(q 34 ; q 11)[4]\end{array}$ & Major & $2^{\text {nd }}$ line \\
\hline 53 & 17 & M & $\mathrm{CP}$ & During course & $46, X, \operatorname{del}(Y),+8, t(9 ; 22)(q 34 ; q 11)[20]$ & Major and minor & $1^{\text {st }}$ line \\
\hline 54 & 18 & M & $\mathrm{CP}$ & At diagnosis & $46, \mathrm{XY}, \mathrm{t}(9 ; 22)(\mathrm{q} 34 ; \mathrm{q} 11)[10] / 59, \mathrm{XY}, \operatorname{der}(22), \mathrm{t}(9 ; 22)(\mathrm{q} 34 ; \mathrm{q} 11)[10]$ & Major & $1^{\text {st }}$ line \\
\hline 55 & 27 & $\mathrm{~F}$ & $A B C$ & At diagnosis & $\begin{array}{l}46, X X,-7,+19, t(9 ; 22)(q 34 ; q 11)[8] / 47, X X,-7,+19,+22, \text { der } \\
(22), t(9 ; 22)(q 34 ; q 11)[4] / 46, X X[8]\end{array}$ & Major and minor & $2^{\text {nd }}$ line \\
\hline 60 & 42 & M & $A B C$ & During course & $\begin{array}{l}46, X Y,-7,+22, \operatorname{der}(22), t(9 ; 22)(q 34 ; q 11)[12] / 45, X Y,-7, t(9 ; 22) \\
(q 34 ; q 11)[8]\end{array}$ & Major and minor & $1^{\text {st }}$ line \\
\hline 62 & 44 & $\mathrm{~F}$ & AP & At diagnosis & $\begin{array}{l}46, X X,+4,-7,-17, \operatorname{der}(22), t(9 ; 22)(q 34 ; q 11)[15] / 46, X X \\
t(9 ; 22)(q 34 ; q 11)[5]\end{array}$ & Major and Minor & $1^{\text {st }}$ line \\
\hline 64 & 50 & $\mathrm{~F}$ & $\mathrm{CP}$ & At diagnosis & $46, \mathrm{XX}, \mathrm{t}(9 ; 11 ; 22)(\mathrm{q} 34 ; \mathrm{q} 23 ; \mathrm{q} 11)[20]$, Variant Ph ch. & Other & $1^{\text {st }}$ line \\
\hline 65 & 54 & M & $\mathrm{CP}$ & At diagnosis & $45, \mathrm{XY},+13,-17,-20[5] / 46, \mathrm{XY}, \mathrm{t}(9 ; 22)(\mathrm{q} 34 ; \mathrm{q} 11)[15]$ & Major and minor & $1^{\text {st }}$ line \\
\hline 66 & 55 & M & $\mathrm{CP}$ & At diagnosis & $\begin{array}{l}53, \mathrm{XY},+4,+8,+10,+12,+15,+19,+22, \operatorname{der}(22), \mathrm{t}(9 ; 22)(\mathrm{q} 34 ; \mathrm{q} 11) \\
{[18] / 48, \mathrm{XY},+4,+22, \operatorname{der}(22), \mathrm{t}(9 ; 22)(\mathrm{q} 34 ; \mathrm{q} 11)[2]}\end{array}$ & Major & $1^{\text {st }}$ line \\
\hline 67 & 23 & $\mathrm{~F}$ & $A B C$ & At diagnosis & $\begin{array}{l}47, \mathrm{XX}, \mathrm{t}(1 ; 3), \mathrm{t}(14 ; 21), \mathrm{t}(16 ; 21),+22, \operatorname{der}(22), \mathrm{t}(9 ; 22)(\mathrm{q} 34 ; \mathrm{q} 11) \\
{[20](\text { complex karyotype) }}\end{array}$ & Major & $1^{\text {st }}$ line \\
\hline 69 & 27 & M & AP & During course & $\begin{array}{l}50, X Y,+4,+8,+10,+22, \operatorname{der}(22), t(9 ; 22)(q 34 ; q 11)[18] / 46, X Y, \\
t(9 ; 22)(q 34 ; q 11)[2]\end{array}$ & Major & $1^{\text {st }}$ line \\
\hline 72 & 31 & $\mathrm{~F}$ & $\mathrm{CP}$ & At diagnosis & $46, X X, \operatorname{der}(1 q), t(9 ; 22)(q 34 ; q 11)[20]$ & Other & $1^{\text {st }}$ line \\
\hline
\end{tabular}




\section{Emergence of ACAs and CML patient's} characteristics

AP patients developed ACAs at a higher percentage $(13 / 21,62 \%)$ compared to CP patients $(19 / 52,36 \%)(p=0.048)$. Patients $<40$ years had a higher incidence of developing ACAs than patients $\geq 40$ years $(p=0.034)$. A higher percentage of patients with normo/hypocellular BM smears $(7 / 8,87 \%)$ developed ACAs compared to $(25 / 65,38 \%)$ with hypercellular BM at diagnosis $(p=0.018)$. A higher percentage of patients with splenomegaly at diagnosis $(31 / 64,48 \%)$ developed ACAs compared to $(1 / 9,11 \%)$ patients with no splenomegaly $(p=0.035)$.

\section{ACAs and TKI response}

CML patients who failed to achieve EMR had more ACAs $(6 / 13,46 \%)$ compared to $(6 / 17,35 \%)$ patients who achieved EMR $(p=0.547)$. A higher percentage of CML-CP patients $(n=52)$ who achieved an optimal molecular response were free of ACAs at diagnosis $(13 / 18,72 \%)$ compared to CP patients with ACAs at diagnosis $(5 / 18,28 \%)(p=0.063)$. We also noted that $C M L-C P$ patients who were free of ACAs at diagnosis were tolerant to their received TKIs $(20 / 34,83 \%)$ compared to patients with ACAs $(14 / 34,41 \%)(p=0.316)$.

\section{OS and EFS in relation to ACAs}

CML patients free from ACAs $(41 / 73,56 \%)$ had a significantly higher median OS (120 vs. 38 months) than those who developed ACAs (32/73, $44 \%)$ at diagnosis or during disease course $(p=0.026)$ (Figure 1).

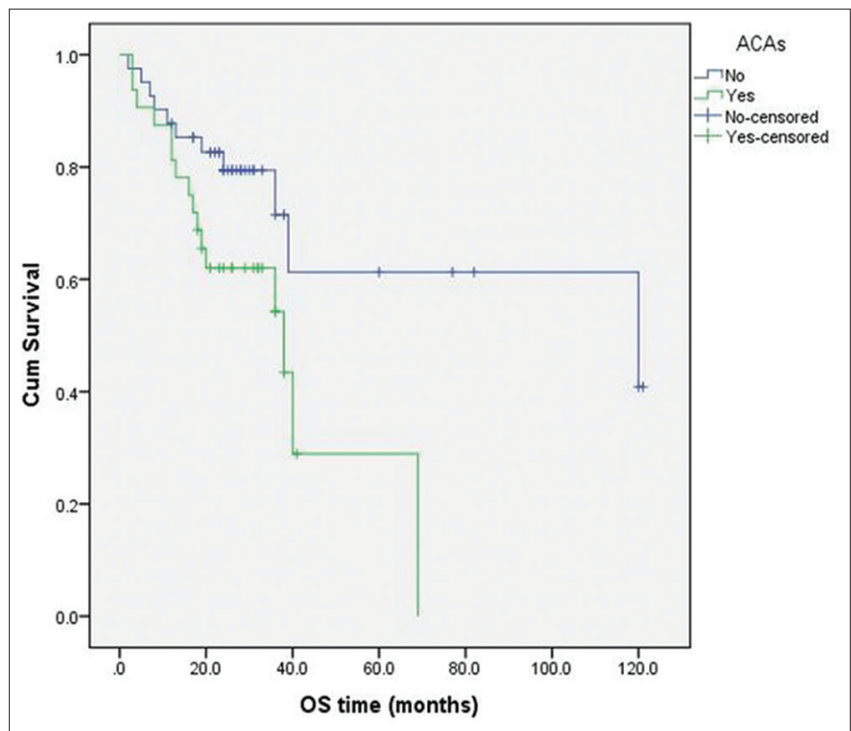

Figure 1: Additional chromosomal abnormalities and overall survival $(p=0.026)$

Patients with no ACAs (41/73, 56\%) had a higher median OS than patients with major route abnormalities (23/73, 31\%) (120 vs. 40 months) in

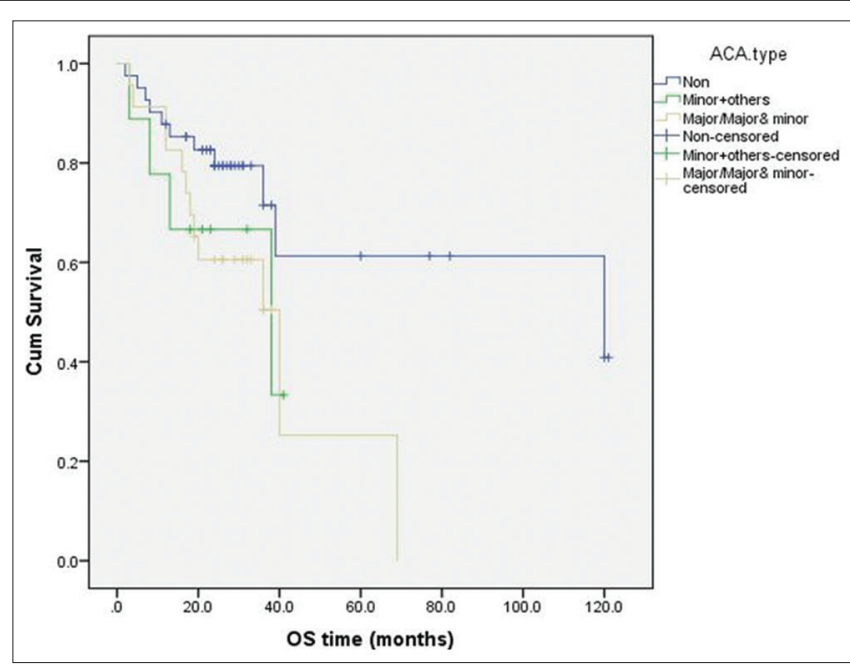

Figure 2: Overall survival by additional chromosomal abnormalities category $(p=0.084)$

pairwise comparison analysis $(p=0.084)$ (Figure 2). CML-CP patients $(n=52)$ who were free of major route ACAs by end of follow-up time $(33 / 52,63 \%)$ had a superior cumulative EFS at 24 months than patients who developed major route abnormalities (12/52, 23\%) $(77 \%$ vs. $58.3 \%)(p=0.065)$ (Figure 3$)$.

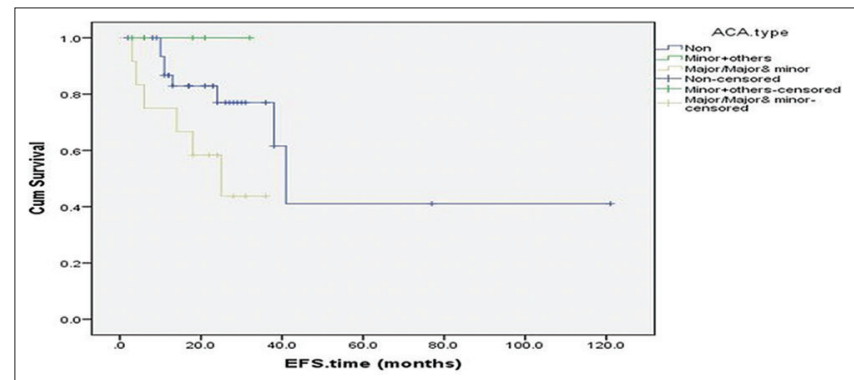

Figure 3: Relationship between additional chromosomal abnormalities category and event-free survival $(p=0.065)$

\section{Correlations between TKI response and patients survival}

The patterns of molecular response and its relation to OS and EFS in $52 \mathrm{CML}-\mathrm{CP}$ patients are described in Table 2. We also report that CML-CP patients $(n=52)$ who shifted to generic IM had a higher cumulative OS ( $85 \%$ vs. $66 \%$ ) and EFS ( $88 \%$ vs. $66 \%)$ at 24 months than patients who had frequent treatment interruptions due to unavailability of $\operatorname{Glivec}^{\circledR}(p=0.087$ and $p=0.100$, respectively).

\section{Correlations between patients characteristics and OS and EFS}

Female patients had a higher median OS than males $(p=0.033)$. CML-CP patients had a higher cumulative survival at 24 months $(84 \%)$ than patients presenting with CML-AP $(42 \%)(p<0.001)$ (Figure 4).

Patients presenting with thrombocytopenia had a lower median OS (20 months) than patients 


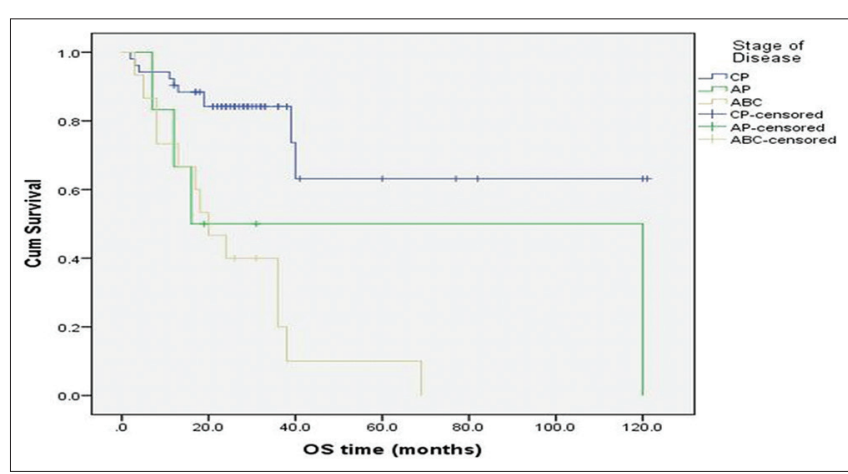

Figure 4: Overall survival according to chronic myeloid leukemia phases at presentation $(p<0.001)$

who presented with higher platelet counts (40 months) $(p=0.070)$. Patients with normo/hypocellular BM had a lower median OS than patients presenting with hypercellular BM (36 vs. 69 months) $(p=0.074)$. Patients with BM eosinophilia $(\geq 5 \%)$ had a higher median OS than patients with BM eosinophils count $<5 \%$ (36 vs. 69 months) $(p=0.01)$. OS was higher in patients with splenomegaly $(73 \%)(p=0.978)$ and hepatomegaly $(85 \%)(p=0.079)$ compared to their normal counterparts (67\% and $60 \%$, respectively). Table 3 illustrates the subgroups of variables considered for the OS comparison between CP and AP groups of CML patients. patients

\section{Predictors of survival in Egyptian CML}

Multivariate analysis of CML patients categorical variables included factors affecting OS in univariate analysis (gender, thrombocytopenia [platelets count $\left.\left.<100 \times 10^{9} / \mathrm{L}\right]\right)$, BM cellularity, BM eosinophilia $(\geq 5 \%)$, disease stage (CP vs. AP), presence of ACAs, and presence of hepatomegaly). Factors that were predictors of OS were the phase of CML disease, BM eosinophilia, and hepatomegaly. AP patients $(n=21)$ were 7 times more liable to death than CP patients $(n=52)(H R=6.9795 \%$, Cl: 3.03-15.96, $p<0.001$ ), patients with no hepatomegaly (39/73) were 2.8 times more liable to death than patients with hepatomegaly $(33 / 73)(\mathrm{HR}=2.8,95 \% \mathrm{Cl}: 1.20$ $6.52, p=0.017)$, and a finding that denotes presence of hepatomegaly was protective in the studied CML group and patients with no BM eosinophilia ( $\geq 5 \%$ ) were 2.37 times more liable to death than patients with BM eosinophilia $(\mathrm{HR}=2.37,95 \% \mathrm{Cl}: 1.053-5.376, \mathrm{p}=0.037)$

\section{Discussion}

Mechanisms of resistance to TKls in CML include genomic amplifications, point mutations of the

Table 2: Patterns of molecular responses and OS and EFS in 52 CML-CP patients

\begin{tabular}{|c|c|c|c|c|c|c|c|}
\hline Type of molecular response & Frequency (\%) & $\begin{array}{l}\text { Major route ACAs } \\
(n=10 / 52)(\%)\end{array}$ & $\begin{array}{l}\text { ACAs }(n=19 / 52) \\
\text { n. }(\%)\end{array}$ & $\begin{array}{l}\text { Cum OS at } \\
24 \mathrm{~ms}(\%)\end{array}$ & $p$ & $\begin{array}{l}\text { Cum EFS at } \\
24 \mathrm{~ms}(\%)\end{array}$ & $\mathrm{p}$ \\
\hline \multicolumn{8}{|l|}{ EMR (3 months) BCR-ABL1 ratio: $(\mathrm{n}=52)$} \\
\hline$<10 \%$ (optimal response) & $30(58 \%)$ & $3(30 \%)$ & $10(33 \%)$ & 76 & \multirow[t]{2}{*}{0.656} & 90 & \multirow[t]{2}{*}{0.051} \\
\hline$\geq 10 \%$ & $22(42 \%)$ & $7(78 \%)$ & $9(41 \%)$ & 77 & & 61 & \\
\hline \multicolumn{8}{|l|}{6 months $B C R-A B L 1$ ratio: $(\mathrm{n}=52)$} \\
\hline$<1 \%$ (optimal response) & $36(68 \%)$ & $6(46 \%)$ & $13(36 \%)$ & 92.3 & \multirow[t]{2}{*}{0.204} & 89 & \multirow[t]{2}{*}{0.049} \\
\hline$\geq 1 \%$ & $16(31 \%)$ & $4(57 \%)$ & $7(44 \%)$ & 83.3 & & 70 & \\
\hline \multicolumn{8}{|l|}{ MMR (12 months) BCR-ABL1 ratio: (n=52) } \\
\hline$<0.1 \%$ (optimal response) & $25(48 \%)$ & $3(37 \%)$ & $8(32 \%)$ & 95 & \multirow[t]{2}{*}{0.276} & 89 & \multirow[t]{2}{*}{0.241} \\
\hline$\geq 0.1 \%$ & $27(52 \%)$ & $7(64 \%)$ & $11(41 \%)$ & 90.5 & & 65 & \\
\hline \multicolumn{8}{|l|}{ DMR (18 months) BCR/ABL1 ratio: (n=52) } \\
\hline$<0.01 \%$ (optimal response) & $20(38 \%)$ & $4(67 \%)$ & $6(30 \%)$ & NR & \multirow[t]{2}{*}{0.424} & 89 & \multirow[t]{2}{*}{0.244} \\
\hline$\geq 0.01 \%$ & $32(61 \%)$ & $6(46 \%)$ & $13(40 \%)$ & NR & & 60 & \\
\hline \multicolumn{8}{|l|}{ At 24 months ( $n=52)$ : } \\
\hline$<0.0001 \%$ (undetectable $B C R-A B L 1$ ratio) (optimal response) & $12(23 \%)$ & $4(100 \%)$ & $4(33 \%)$ & NR & \multirow[t]{2}{*}{ NR } & 67 & \multirow[t]{2}{*}{ NR } \\
\hline$\geq 0.0001 \%$ & $40(80 \%)$ & $6(40 \%)$ & $15(37 \%)$ & NR & & 83 & \\
\hline \multicolumn{8}{|l|}{ Type of response to TKI therapy: } \\
\hline Optimal & $18(35 \%)$ & $3(60 \%)$ & $5(28 \%)$ & 94.4 & \multirow[t]{3}{*}{0.125} & 94 & \multirow[t]{3}{*}{0.065} \\
\hline Suboptimal (warning ) & $10(19 \%)$ & $2(50 \%)$ & $4(40 \%)$ & NR & & 79 & \\
\hline Failure & $24(46 \%)$ & $5(50 \%)$ & $10(42 \%)$ & 70.8 & & 57 & \\
\hline
\end{tabular}

OS: Overall survival, EFS: Event-free survival, TKI: Tyrosine kinase inhibitors.

Table 3: Predictors of OS in CML-CP and AP phases of CML

\begin{tabular}{|c|c|c|c|c|c|c|c|c|c|c|c|}
\hline \multirow{2}{*}{$\begin{array}{l}\text { Parameter } \\
\text { Totals }\end{array}$} & \multicolumn{2}{|l|}{$\mathrm{n}(\%)$} & \multicolumn{2}{|c|}{ No of deaths (\%) } & \multicolumn{2}{|c|}{ Cum survival at 12 months (\%) } & \multicolumn{2}{|c|}{ Cum. survival at 24 months (\%) } & \multicolumn{2}{|c|}{ Median survival (months) } & \multirow[t]{3}{*}{$\mathrm{p}$} \\
\hline & $\mathrm{CP}$ & AP & $\mathrm{CP}$ & AP & $\mathrm{CP}$ & AP & $\mathrm{CP}$ & AP & $\mathrm{CP}$ & $\mathrm{AP}$ & \\
\hline & 52 & 21 & $10(19 \%)$ & $17(81 \%)$ & 90 & 71 & 84 & 42 & * & 20 & \\
\hline \multicolumn{12}{|l|}{ Age (years) } \\
\hline$<40$ & $26(50)$ & $14(67)$ & $3(11)$ & $11(79)$ & 92 & 71 & 88 & 36 & * & 18 & $<0.001$ \\
\hline$\geq 40$ & $26(50)$ & $7(33)$ & $7(27)$ & $6(86)$ & 89 & 71 & 80 & 57 & * & 36 & 0.066 \\
\hline \multicolumn{12}{|l|}{ Gender } \\
\hline Male & $22(42)$ & $9(43)$ & $7(32)$ & $9(100)$ & 86 & 78 & 71 & 22.2 & 40 & 17 & 0.004 \\
\hline Female & $30(58)$ & $12(57)$ & $3(10)$ & $8(67)$ & 93 & 67 & * & 57.1 & * & 36 & $<0.001$ \\
\hline \multicolumn{12}{|l|}{ ACAs } \\
\hline Present & $19(36)$ & $13(62)$ & $6(32)$ & $10(77)$ & 84 & 77 & 73 & 45 & 40 & 20 & 0.075 \\
\hline Absent & $33(63)$ & $8(38)$ & $4(12)$ & $7(87)$ & 94 & 63 & 91 & 37 & * & 13 & $<0.001$ \\
\hline \multicolumn{12}{|l|}{ ACAs category } \\
\hline None & $33(63)$ & $8(38)$ & $4(12)$ & $7(87)$ & 94 & 63 & 91 & 37 & * & 13 & $<0.001$ \\
\hline $\begin{array}{l}\text { Major + (major and } \\
\text { minor). }\end{array}$ & $12(23)$ & $11(52)$ & $4(33)$ & $8(73)$ & 83 & 81 & 75 & 44 & 40 & 20 & 0.172 \\
\hline Minor + others & $7(14)$ & $2(10)$ & $2(29)$ & $2(29)$ & 86 & 50 & 71 & * & * & 8 & -.--- \\
\hline \multicolumn{12}{|l|}{ Hepatomegaly } \\
\hline No & $28(54)$ & $11(52)$ & $7(25)$ & $10(91)$ & 86 & 45 & 78 & 18 & * & 12 & $<0.001$ \\
\hline Yes & $24(46)$ & $10(48)$ & $3(13)$ & $7(70)$ & 96 & * & 92 & 69 & * & 38 & 0.019 \\
\hline
\end{tabular}


$A B L$ tyrosine kinase domain, and emergence of ACAs [15]. The median age of patients at diagnosis was 37 years which is comparable to previous studies in Pakistan, Nigeria, Mexico [16], [17], [18], and previous Egyptian reports [19], [20]. In our study, we found that patients $<40$ years tended to develop ACAs more than patients $\geq 40$ years $(55 \%$ vs. $30 \%)$, respectively $(p=0.034)$. Similarly, median age (27 years) of patients who developed trisomy 8 , a major route abnormality was higher than the median age of patients (40 years) who were free from the same abnormality $(p=0.023)$. The same observation was detected when comparing the median age of CML patients with an extra Philadelphia chromosome (17/73, 32 years) and without the same abnormality (56/73) (38 years) ( $p=0.225)$. These findings matched those of GIMEMA working group who reported a lower median age of 45 years in patients with ACAs compared to 50 years in patients without ACAs [21]. Our findings also matched those reported by the German CML study Group IV who reported a lower median age of 48 years in patients with ACAs compared to 53 years in patients with the sole standard $t(9 ; 22)$ abnormality [22]. In our study, the incidence of ACAs was 44\% (32/73) and this was higher than the highest previously reported incidence by others (15\%, 6\%, 7\%, and 24\%), respectively [16], [21], [22], [23]. In addition, the incidence of major route abnormalities was $18 / 73,25 \%$. Our observed incidence was comparable to two other reports showing an ACAs incidence of $42 \%$ and $30 \%$, respectively [24], [25]. Incidence of emergence of ACAs in our CML-CP patients was $36 \%$ and $62 \%$ in AP ( $p=0.048)$. These findings were in agreement with previously reported data by others [23], [26]. We found that a significantly higher percentage of patients with splenomegaly (48\%) developed ACAs during their disease course compared to $11 \%$ of patients with no splenomegaly ( $p=0.035$ ). This finding was in accordance with a previous study [21], who reported a higher median increased spleen size $(4 \mathrm{~cm})$ in patients with ACAs compared to a median of $2 \mathrm{~cm}$ in those without ACAs. This finding could be explained by the fact that CML patients with a higher Sokal prognostic score at diagnosis have a higher probability to develop ACAs [13]. We also describe an association between hepatomegaly at presentation and a higher median OS ( $p=0.086)$ in univariate survival analysis as the presence of hepatomegaly was found to be protective and a favorable independent predictor of OS in the multivariate model $(\mathrm{HR}=2.80,95 \% \mathrm{Cl}: 1.20$ $6.52, p=0.017$ ). The protective role of hepatomegaly could be explained by the fact that liver represents an extramedullary site of hematopoiesis that takes over this process in the event of BM infiltration by myeloid elements as observed in $\mathrm{CML}$, thus maintaining other blood lineages physiological counts and providing tumor infiltrating lymphocytes to carry on an effective antitumor immune response. In this work, CML patients with hypercellular BM smears at presentation developed less ACAs than those who presented with a normal or reduced BM cellularity $(p=0.018)$, explained in light of the presence of concomitant BM fibrosis in patients with reduced BM cellularity at the presentation which would classify these patients as an advanced disease (AP) CML even before any evidence of $\mathrm{CE}$, an explanation confirmed by BM trephine specimens examination of included $\mathrm{CML}$ patients [8]. BM basophils $<3 \%$ in our CML-CP patients $(n=52)$ was significantly associated with a higher median EFS than patients with BM basophils $\geq 3 \%(p=0.049)$. This finding was in agreement with that reported by Cortes et al., 2003 [23], who used a $5 \%$ cutoff for BM basophils and observed the same relationship between the two groups of CP patients and achieving MCyR. However, we found a different relationship between $B M$ eosinophilia $(\geq 5 \%)$ and OS in our Egyptian CML patients describing eosinophilia to be a favorable prognostic factor and independent predictor of survival in Egyptian CML patients upon univariate $(p=0.014)$ and multivariate analyses models $(p=0.037$, $\mathrm{HR}=2.38,95 \% \mathrm{Cl}=1.05-5.38)$. This was different to what was reported by the Hasford and EUTOS scoring systems to evaluate risk groups in CML patients [27]. The mentioned scoring systems utilize PB eosinophils percentage in its formula, denoting that a higher $\mathrm{PB}$ eosinophils \% results in a higher patient score and higher risk category. Although PB eosinophils percentage and not BM eosinophils are included in Hasford and EUTOS scoring systems [27], the current finding in this study introduces BM eosinophils percentage as an independent predictor of OS in Egyptian CML patients and a variable that could be potentially introduced in risk stratification of CML in middle-Eastern populations, although larger cohort studies are needed to justify this attempt. Patients with thrombocytopenia $(<100 \times 109 / L)$ had a lower median OS than patients who presented with higher platelet counts $(p=0.07)$ and was found to be an independent poor prognostic factor in Egyptian CML patients upon the log-rank statistical test of the impact of variables on OS $(p=0.082)$. This correlation was similar to a previously reported significant correlation between thrombocytopenia at presentation and unresponsiveness to TKI therapy and lack of MCyR which was consequently and significantly correlated with OS and EFS [28]. A higher percentage of CML-CP patients who failed to achieve optimal response to TKI therapy had ACAs (42\%) compared to $(28 \%)$ of optimal responders. These findings were comparable to reported findings by Cortes et al., 2003, who concluded that either CP or AP CML patients who developed ACAs had inferior hematologic and cytogenetic responses to TKI than their ACAs free counterparts. Our presented findings also matched those of the GIMEMA working party who reported a lower percentage of optimal responders (major molecular response at 12 months) among patients with ACAs (67\%) compared to $86 \%$ among patients without ACAs. In the current work, median OS of CP patients at the end of 24 months follow-up duration could not be reached as more than half of patients were still alive and median survival was 
21 months for AP patients. As expected, the presenting phase of CML at diagnosis was found to be an independent poor prognostic factor in univariate and multivariate analyses with CML-CP patients having a significantly higher cumulative OS at 24 months than patients with AP $(\mathrm{p}<0.001)(\mathrm{HR}=7,95 \% \mathrm{Cl}=3.03-$ 15.9, $p<0.001)$.We also found that $C M L$ patients who were free of ACAs had a significantly higher OS than patients who developed ACAs $(p=0.026)$ and a higher cumulative EFS at 24 months $(p=0.065)$. This finding was in accordance with that reported by others [21], [23]. We were able to identify a negative impact of major route ACAs in a total of 18 patients (11 in CP and 8 in AP of $\mathrm{CML}$ ) on OS and EFS compared to patients with the minor route or other abnormalities $(p=0.084$ and $p=0.065)$, respectively. We also attempted to evaluate the relationship between the type of TKI received upfront whether $1^{\text {st }}$ line $(\mathrm{IM})$ or $2^{\text {nd }}$ line (Nilotinib and Dasatinib), time to TKI treatment and shift to generic IM and the frequency of developing ACAs, molecular response, patient's tolerance to TKIs, and their OS and EFS. We found that among patients who developed ACAs $(n=32)$, a larger number of patients had a $>15$ days' time to $\operatorname{TKI}(25 / 30,83 \%)$ compared to $(5 / 30,17 \%)$ with shorter time to TKI ( $\leq 15$ days) $(p=0.130)$. This finding necessitates earlier therapy from time of diagnosis to eliminate CE. We also found that a larger number of patients who received $1^{\text {st }}$ line TKI upfront tended to develop ACAs (30/32, 94\%) compared to those who received $2^{\text {nd }}$ line $\mathrm{TKI}$ as an upfront therapy (2/32, 6\%), a finding proving a deeper molecular response achieved by $2^{\text {nd }}$ line TKI, affecting survival and elimination of CE. In addition, a larger number of patients who had interruptions to Glivec ${ }^{\circledR}$ therapy without shifting to other generic IM (23/32, 72\%) developed ACAs compared to those who shifted to a different generic IM without treatment interruption (9/32, 28\%). We also report that CP patients who were tolerant to their received TKIs tended to develop ACAs less $(14 / 34,17 \%)$ than their counterparts who were defined as intolerant to TKIs $(2 / 6,33 \%)$. These observations could be matched with previous studies [10], [28], [29]. An interesting observation that we report in this study is the near significance higher median OS and EFS of CML-CP patients who had a shift to a different generic IM during their treatment course due to the unavailability of Glivec ${ }^{\circledR}$ ( $p=0.087$ and $p=0.100$, respectively). However, due to the small number of patients in the crossover to generic IM group ( $n=22)$ compared to their counterparts who did not receive the same generic drug $(n=43)$, we could not reach a statistically significant difference.

\section{Conclusion}

We report a higher incidence of ACAs in our Egyptian CML patients at diagnosis and during the course of therapy than western populations. The appearance of ACAs, particularly, major route abnormalities at diagnosis or their emergence during treatment plays an important role in defining response to TKI therapy and have a negative impact on CML patients response to TKI therapy in terms of cytogenetic and molecular responses as a major mechanism of resistance to TKI therapy; this finding also applies to $\mathrm{CML}$ patients presenting in $\mathrm{AP}$ of the disease. CML patients who developed ACAs, particularly major route abnormalities, had a lower OS and EFS than their counterparts without ACAs, which proves the negative impact of $C E$ in this disease. We conclude that the impact of ACAs on response to TKIs therapy in CML patients has important clinical and biological implications. Our study suggests that the detection of ACAs, besides $B C R-A B L 1$ kinase domain mutation, is an important determinant of CML patients prognosis and survival. Earlier identification of these abnormalities may help in adopting more appropriate therapeutic approach.

\section{Acknowledgments}

We thank our colleagues, staff, and Dr. Basma M. Elgamal (head of cytogenetics unit) at National Cancer Institute of Cairo University for their expert technical assistance.

\section{References}

1. Jabbour $E$, Kantarjian $H$. Introduction: Chronic myelogenous leukemia (CML). Semin Hematol 2007;44(1 Suppl 1):S1-3. https://doi.org/10.1182/blood-2008-03-144790

PMid:17292735

2. Quintas-Cardama A, Cortes J. Molecular biology of bcr-abl1positive chronic myeloid leukemia. Blood 2009;113(8):1619-30. PMid:18827185

3. Marktel S, Marin D, Foot N, Szydlo R, Bua M, Karadimitris A, et al. Chronic myeloid leukemia in chronic phase responding to imatinib: The occurrence of additional cytogenetic abnormalities predicts disease progression. Haematologica 2003;88(3):260-7. PMid:12651263

4. Mitelman F. The cytogenetic scenario of chronic myeloid leukemia. Leuk Lymphoma 1993;11(Suppl 1):11-5. https://doi. org/10.3109/10428199309047856

PMid:8251885

5. Baccarani M, Saglio G, Goldman J, Hochhaus A, Simonsson B, Appelbaum F, et al. Evolving concepts in the management of chronic myeloid leukemia: Recommendations from an expert panel on behalf of the European LeukemiaNet. Blood 2006;108(6):180920. https://doi.org/10.1182/blood-2006-02-005686 PMid:16709930

6. de Lavallade H, Apperley JF, Khorashad JS, Milojkovic D, Reid AG, Bua M, et al. Imatinib for newly diagnosed patients with chronic myeloid leukemia: Incidence of sustained responses in 
an intention to-treat analysis. J Clin Oncol 2008;26(20):3358. https://doi.org/10.1200/jco.2007.15.8154

PMid:18519952

7. O'Brien SG, Guilhot F, Larson RA, Gathmann I, Baccarani M, Cervantes $\mathrm{F}$, et al. Imatinib compared with interferon and lowdose cytarabine for newly diagnosed chronic-phase chronic myeloid leukemia. N Engl J Med 2003;348(11):994-1004. https://doi.org/10.1056/nejmoa022457

8. Swerdlow S, Campo E, Harris NL, Jaffe ES, Pileri SA, Stein H, et al. WHO Classification of Tumours of Haematopoietic and Lymphoid Tissues. $4^{\text {th }}$ ed. Geneva: World Health Organization; 2016.

9. Cortes JE, Talpaz M, O'Brien S, Faderl S, Garcia-Manero G, Ferrajoli A, et al. Staging of chronic myeloid leukemia in the imatinib era: An evaluation of the World Health Organization proposal. Cancer 2006;106(6):1306-15. https://doi.org/10.1002/cncr.21756 PMid:16463391

10. Baccarani M, Cortes J, Pane F, Niederwieser D, Saglio G, Apperley J, et al. Chronic myeloid leukemia: An update of concepts and management recommendations of European LeukemiaNet. J Clin Oncol 2009;27(35):6041-51. https://doi. org/10.1200/jco.2009.25.0779

PMid:1988452

11. National Comprehensive Cancer Network. NCCN Practice Guidelines in Oncology. Vol. 3. United States: National Comprehensive Cancer Network; 2020. Available from: http:// www.nccn.org/professionals/physician_gls/pdf/cml.pdf. https:// doi.org/10.1158/1538-7755.disp19-d069.

12. Baccarani M, Deininger MW, Rosti G, Hochhaus A, Soverini S, Apperley JF, et al. European leukemia net recommendations for the management of chronic myeloid leukemia. Blood 2013;122(6):872-84 .

PMid:23803709

13. Press RD, Love Z, Tronnes AA, Yang R, Tran T, MongoueTchokote $\mathrm{S}$, et al. BCR-ABL mRNA levels at and after the time of a complete cytogenetic response (CCyR) predict the duration of CCyR in imatinib mesylate treated patients with CML. Blood 2006;107:4250-6. https://doi.org/10.1182/blood-2005-11-4406 PMid:16467199

14. Jean MJ, Simons A, Schmid M. ISCN, 2016: An International System for Human Cytogenomic Nomenclature. Basel, Switzerland: Karger; 2016. https://doi.org/10.1159/ isbn.978-3-318-05979-3

15. Hochhaus A. Cytogenetic and molecular mechanisms of resistance to imatinib. Semin Hematol 2003;40(Suppl 2):69-79. PMid: 12783379

16. Naveen S, Mohammad U, Salman A, Mohammad K. Additional chromosomal abnormalities in philadelphia-positive chronic myeloid leukemia. Hematol Oncol Stem Cell Ther 2008;3:16670. https://doi.org/10.1016/s1658-3876(08)50025-2 PMid:20063547

17. Oyekunle AA, Bolarinwa RA, Oyelese AT, Salawu L, Durosinmi MA. Determinants of overall and progression-free survival of Nigerian patients with Philadelphia-positive chronic myeloid leukemia. Adv Hematol 2015;2015:908708. https://doi. org/10.1155/2015/908708

PMid:26435715

18. Ylescas-Soria J, de la Torre-Lujan AH, Montalvo LA, Miranda D, Grimaldo F, Rivas S, et al. Prognostic factors for overall survival in patients with chronic myeloid leukemia treated with imatinib at the National Cancer Institute Mexico, from 2000 to 2016. Cancer Med 2019;8(6):2942-9. https://doi.org/10.1002/cam4.2201 PMid:31050162

19. Elnahass YH, Mahmoud HK, Ali FT, Mohamed MR, Said MM,
Samra MA, et al. Abl kinase domain mutations in imatinib-treated Egyptian patients with chronic myeloid leukemia. J Leukemia 2013;1(1):1-7. https://doi.org/10.4172/2329-6917.1000106

20. El-Metnawy $W$, Mattar M, El-Nahass $Y$, Samra MA, Abdelhamid HM, Abdlfattah RM, et al. Predictive Value of Pretreatment BCR-ABL Transcript level on Response to Imatinib Therapy in Egyptian Patients with Chronic Phase Chronic Myeloid Leukemia (CPCML). Int J Biomed Sci 2013;9(1):48-53. https://doi.org/10.4172/2329-6917.1000106 PMid:23675289

21. Luatti S, Castagnetti F, Marzocchi G, Baldazzi C, Gugliotta G, lacobucci I, et al. Additional chromosome abnormalities in Philadelphia positive clone: Adverse prognostic influence on frontline imatinib therapy: A GIMEMA Working Party on CML analysis. Blood 2012;120:761-7. https://doi.org/10.1182/ blood-2013-05-502054

22. Fabarius A, Leitner A, Hochhaus A, Müller MC, Hanfstein B, Haferlach $\mathrm{C}$, et al. Impact of additional cytogenetic aberrations at diagnosis on prognosis of CML: Long-term observation of 1151 patients from the randomized CML Study IV. Blood 2011;118(26):6760-8. https://doi.org/10.1182/blood.v118.21.782.782 PMid:22039253

23. Cortes JE, Talpaz M, Giles F, O'Brien S, Rios MB, Shan J, et al. Prognostic significance of cytogenetic clonal evolution in patients with chronic myelogenous leukemia on imatinib mesylate therapy. Blood 2003;101(10):3794-800. https://doi. org/10.1182/blood-2002-09-2790 PMid:12560227

24. Mohamed AN, Pemberton P, Zonder J, Schiffer CA. The effect of imatinib mesylate on patients with Philadelphia chromosomepositive chronic myeloid leukemia with secondary chromosomal aberrations. Clin Cancer Res 2003;9(4):1333-7. PMid:12684401

25. Wang W, Cortes JE, Tang G, Khoury JD, Wang S, BuesoRamos CE, et al. Risk stratification of chromosomal abnormalities in chronic myelogenous leukemia in the era of tyrosine kinase inhibitor therapy. Blood 2016;127(22):2742-50. https://doi.org/10.1182/blood-2016-01-690230 PMid:27006386

26. Haferlack C, Bacher U, Schnittger S, Weiss T, Kern W, Haferlach T. Similar patterns of chromosome abnormalities in CML occur in addition to the Philadelphia chromosome with or without tyrosine kinase inhibitor treatment. Leukemia 2010;24(3):638-40. https://doi.org/10.1038/leu.2009.222 PMid:19865111

27. Hasford J, Baccarani M, Hoffmann V, Guilhot J, Saussele S, Rosti G, et al. Predicting complete cytogenetic response and subsequent progression-free survival in 2060 patients with CML on imatinib treatment: The EUTOS score. Blood 2011;118(3):68692. https://doi.org/10.1182/blood-2010-12-319038 PMid:21536864

28. Furtado VF, Santos GR, de Carvalho DS, Staziaki PV, Pasquini R, Funke VA. Accelerated phase chronic myeloid leukemia: Evaluation of clinical criteria as predictors of survival, major cytogenetic response and progression to blast phase. Rev Bras Hematol Hemoter 2015;37(5):341-7. https://doi. org/10.1016/j.bjhh.2015.07.004 PMid:26408370

29. Pinilla-Ibarz J, Cortes J, Mauro MJ. Intolerance to tyrosine kinase inhibitors in chronic myeloid leukemia: Definitions and clinical implications. Cancer 2011;117(4):688-97. https://doi. org/10.1002/cncr.25648

PMid:20922786 